\title{
BMJ Open Does geriatric follow-up visits reduce hospital readmission among older patients discharged to temporary care at a skilled nursing facility: a before-and- after cohort study
}

\author{
Katja Thomsen (D) , ${ }^{1,2}$ Anders Fournaise (D) ,,3 Lars Erik Matzen, ${ }^{1,2}$ \\ Karen Andersen-Ranberg, ${ }^{1,4}$ Jesper Ryg (D) ${ }^{1,2}$
}

To cite: Thomsen $\mathrm{K}$,

Fournaise A, Matzen LE, et al. Does geriatric follow-up visits reduce hospital readmission among older patients discharged to temporary care at a skilled nursing facility: a before-andafter cohort study. BMJ Open 2021;11:e046698. doi:10.1136/ bmjopen-2020-046698

- Prepublication history for this paper is available online. To view these files, please visit the journal online (http://dx.doi. org/10.1136/bmjopen-2020046698).

$\mathrm{KT}$ and $\mathrm{AF}$ are joint first authors.

Received 06 November 2020 Accepted 22 July 2021

Check for updates

(C) Author(s) (or their employer(s)) 2021. Re-use permitted under CC BY-NC. No commercial re-use. See rights and permissions. Published by BMJ.

For numbered affiliations see end of article.

Correspondence to

Dr Katja Thomsen;

katja.thomsen@rsyd.dk

\section{ABSTRACT}

Introduction Hospital readmission is a burden to patients, relatives and society. Older patients with frailty are at highest risk of readmission and its negative outcomes.

Objective We aimed at examining whether follow-up visits by an outgoing multidisciplinary geriatric team (OGT) reduces unplanned hospital readmission in patients discharged to a skilled nursing facility (SNF).

Design A retrospective single-centre before-and-after cohort study.

Setting and participants Study population included all hospitalised patients discharged from a Danish geriatric department to an SNF during 1 January 2016-25 February 2020. To address potential changes in discharge and readmission patterns during the study period, patients discharged from the same geriatric department to own home were also assessed.

Intervention OGT visits at SNF within 7 days following discharge. Patients discharged to SNF before 12 March 2018 did not receive OGT (-OGT). Patients discharged to SNF on or after 12 March 2018 received the intervention (+OGT).

Main outcome measures Unplanned hospital readmission between 4 hours and 30 days following initial discharge.

Results Totally 847 patients were included (440 -0GT; $407+$ OGT). No differences were seen between the two groups regarding age, sex, activities of daily living (ADLs), Charlson Comorbidity Index (CCI) or 30-day mortality. The cumulative incidence of readmission was $39.8 \%$ (95\% Cl $35.2 \%$ to $44.8 \%, \mathrm{n}=162)$ in -OGT and $30.2 \%(95 \% \mathrm{Cl}$ $25.8 \%$ to $35.2 \%, n=113$ ) in +OGT. The unadjusted risk (HR $(95 \% \mathrm{Cl}))$ of readmission was $0.68(0.54$ to $0.87, \mathrm{p}=0.002)$ in +0GT compared with -0GT, and remained significantly lower $(0.72(0.57$ to $0.93, p=0.011))$ adjusting for age, length of stay, sex, ADL and CCl. For patients discharged to own home the risk of readmission remained unchanged during the study period.

Conclusion Follow-up visits by OGT to patients discharged to temporary care at an SNF significantly reduced 30-day readmission in older patients.
Strengths and limitations of this study

- This was a hospital-based before-and-after cohort study with no patients lost to follow-up.

- We accounted for the competing risk of death and adjusted for potential confounders in our analysis (age, sex, activities of daily living, comorbidity and length of stay).

- We explored general changes in readmission pattern not related to the intervention.

- This was a before-and-after cohort study, which has a risk of overestimating the effect.

- This was a single-centre study, which may limit the generalisability of study results.

\section{INTRODUCTION}

Acute hospitalisation can be life-saving but may also lead to adverse health outcomes in older adults, such as hospital-acquired infections and poorer functional health, as well as anxiety and distress. ${ }^{1-7}$

Some acute hospitalisations are preventable, and particularly readmissions are therefore in focus for preventive initiatives. Readmission risk increases with age, especially in patients characterised by multimorbidity, polypharmacy, longer in-hospital stay, lower functional status, male sex and prior hospitalisation..$^{8-10}$ After an acute treatment, such vulnerable patients may be transferred to a postacute care facility for further stabilisation of medical and functional health, either in a hospital or in a skilled nursing facility (SNF), which offers temporary stays. $\mathrm{SNF}$ is an in-patient rehabilitation centre staffed with nurses and allied health professionals, while medical attention is carried out by a primary care physician.

Interventions to prevent readmission among older adults are widely studied. Several 
models have been investigated; involving various staff groups (ie, pharmacist, nurse, primary care physician and geriatrician) and different point of actions (ie, nutrition, medication, exercise, care), ${ }^{11-14}$ but no single or a bundle of interventions appear to reduce hospital readmission reliably. ${ }^{15-17}$ For patients discharged to an SNF, only few intervention studies exist, ${ }^{4-6}{ }^{18-20}$ mainly from countries with a mixture of public and private healthcare providers.

Our aim was to examine whether early follow-up visits by an outgoing multidisciplinary (nurse and doctor) geriatric team (OGT) reduce acute hospital readmission in older vulnerable patients discharged to a temporary stay in an SNF.

\section{METHODS}

\section{Design}

We conducted a retrospective single-centre, before-andafter cohort study, following the implementation of an OGT at an SNF in the municipality of Odense, Denmark.

\section{Settings}

In Denmark, all citizens have free access to their primary care physician, hospital treatment and healthcare services including home care due to a tax-funded public healthcare system. Hospital treatment is provided by five regions, who also are responsible for financing primary care physicians and specialist physicians, while 98 municipalities are responsible for providing home care, social care, rehabilitation and health promotion as well as permanent and temporary care at SNF. ${ }^{21}$

Odense University Hospital is a highly specialised hospital serving citizens in the Region of Southern Denmark. It is the main hospital for the citizens of Odense municipality with approximately 200000 inhabitants, of which $17 \%$ are above 65 years of age. ${ }^{22}$ The Department of Geriatric Medicine at Odense University Hospital consists of a medical ward with 38 beds. All patients are admitted as acute patients from the emergency department or transferred from other departments, and are characterised by acute medical illness, high age, multimorbidity, polypharmacy and functional decline. Occasionally, the department treats patients with planned admissions, for example, preparation for colonoscopy. All other planned treatments are conducted as ambulant care in the department's outpatient clinical, only $0.5 \%$ were planned admissions during study period. The department has a close collaboration with Odense Municipality, which is responsible for providing home care and nursing care services for patients after hospital discharge. ${ }^{21}$ Most patients $(87 \%)$ are discharged to the same residence they had before admission. Patients admitted to hospital from their own home, who at the time of discharge are in need of substantial care and rehabilitation can be recommended by the hospital to be discharged to a municipal SNF for temporary care and rehabilitation. However, the municipality decides whether the recommendation should be complied with or the patients should be cared for in their own home.

Odense municipality has organised their SNF in one facility hosting 64 temporary beds located $7 \mathrm{kms}$ from Odense University Hospital. The SNF has a turnover of approximately 1000 patients a year with an average length of stay (LOS) of 24 days (unpublished administrative data). The SNF staff includes nurses, social and healthcare assistants, physiotherapists and occupational therapist, but there is no staff physician. If a patient needs medical attention, the SNF staff contacts the patient's personal primary care physician.

\section{Intervention}

The OGT was developed as part of a quality assurance project at Odense University Hospital aiming at reducing unnecessary readmissions among older citizens. The personnel did not follow a prespecified protocol. However, medical staff at the Department of Geriatric Medicine and the personnel at the SNF developed a co-operation agreement specifying the scope of the OGT-visit, mutual responsibilities in the cooperation, and which medical issues to handle during the OGT-visit.

The OGT was initiated 12 March 2018. It consisted of a geriatric nurse and a specialist geriatrician, who in close collaboration with the patient, and the SNF-staff dealt with any health issues related to the recent hospital admission and discharge, that is, effect and adverse effects of initiated treatment, nutritional and rehabilitation issues, and any uncertainties of the discharge summary. The OGT consulted patients at the SNF within 7 days following hospital discharge. The OGT had access to the patients' electronic hospital records, including records from physicians, nurses, occupational and physiotherapists, and an updated list of medications. Moreover, venous blood samples, urine and stool samples could be collected by the OGT for analyses at the hospital. The SNF-staff was instructed to closely read the hospital discharge summary, to pay a special attention to effect and potential adverse effect of initiated treatment, and to nutritional and to rehabilitation issues. Moreover, it was required that blood pressure, pulse, temperature and blood oxygen saturation were measured ahead of OGT visits. The OGT visited the SNF three times a week, totally 6 hours per week. Patients received at least one OGT visit from the OGT during the follow-up.

\section{Participants}

We included all patients discharged from the Department of Geriatric Medicine at Odense University Hospital from 1 January 2016 to 25 February 2020. Eligible patients were identified from Odense University Hospital's patient administrative system and the Odense municipality's electronic care journal. Patients discharged to SNF (SNF patients) before and after initiation of OGT 12 March 2018 were categorised as -OGT and as +OGT, respectively. To explore time trends and potential variations in discharge, readmission patterns and patient characteristics during 
the study period, we categorised patients discharged to own home (non-SNF patients) before and after 12 March 2018 as pre-OGT and post-OGT, respectively.

\section{Variables and data sources}

The primary outcome was 30-day readmission rate. Readmission was defined as any acute/unplanned hospital admission within 4 hours and 30 days after discharge from the Department of Geriatric Medicine. ${ }^{23}$ Index admission was defined as any unplanned admission to the Department of Geriatric Medicine during the study period. Patients with several hospital admissions could have several index admissions but only one readmission per index admission. Thus, each unplanned admission to the geriatric department counted as an index admission.

We used the patient's unique civil registration code to extract data from Odense University Hospitals patient administrative system for each index admission. Data included information of unplanned hospital admissions, date and time of the admission and discharge, location, type and hospital department. Length of index admissions and readmissions were derived from medical records. Participants' characteristics for example, age, sex, and information on mortality were obtained from the Civil Registration System. Information of comorbidity and Barthel index (BI) were obtained from Odense University Hospitals patient administrative system or directly from medical records when missing in the register $(n=59)$. BI is a measure of function in activity of daily living (ADL), with a sum score across ten domains of ADL. BI is the official ADL tool used in Danish hospitals and assessed routinely on hospital admission. ${ }^{24}$ The total score ranges from 0 (completely dependent) to 100 (completely independent). In Denmark BI is categorised in four standard ICD-10 diagnostic categories $\mathrm{BI}=80-100$ (independent $\mathrm{ADL}$ ), $\mathrm{BI}=50-79$ (moderate reduced $\mathrm{ADL}$ ), $\mathrm{BI}=25-49$ (low $\mathrm{ADL}$ ), and $\mathrm{BI}=0-24$ (very low $\mathrm{ADL}$ ). The burden of comorbidity was assessed using Charlson Comorbidity Index (CCI), which was computed based on all primary and secondary discharge diagnoses registered in the hospital's electronic patient journal the past 4 years. The CCI score was divided into three levels: low (score of 0 ), moderate (score of 1-2) and high (score of $\geq 3$ ). ${ }^{25}$

The health interventions by the OGT were registered in an administrative database without any personally identifiable data. This database provided summary data of the interventions made by the OGT (ie, adjustments of medication, blood samples, nutritional advice, information of tests/examinations and intervention from a specialised acute nursing function). Data of number of OGT-visits per patient were extracted from the patient administrative system.

\section{Statistical analysis}

The study sample size was calculated from expected change in readmission rate 'before and after'. The inclusion of 367 patients in each group would give the study $80 \%$ power to detect a $30 \%$ reduction in readmission rate assuming a baseline readmission rate of $30 \%,{ }^{19}$ with a level of significance of $5 \%$. This power calculation was used to define the length of the study period in order to ensure an adequate sample size. Patient characteristics were reported using numbers/percentages, means (SD) and medians (IQR). Differences between groups were calculated using $\chi^{2}$ tests, Student's t-test or Kruskal-Wallis test, as appropriate. The primary outcome, readmission rate, was calculated as the total number of 30 days readmissions divided by total number of index admissions. A statistical process control chart plot was created plotting monthly readmission rates over the entire study period. A Cox proportional hazard model for readmission within 30 days was used to investigate the effect of the OGT. Patients who died or moved to another municipality within 30 days after discharge were censored from analysis and the model was adjusted for age, LOS, sex, CCI and BI. The incidence of readmission was plotted as a function of time in a Kaplan-Meier plot of cumulative incidence. The analysis did not include patients with a follow-up of less than 12 hours. The proportional-hazard assumption was tested using Schoenfeld residuals. The statistical significance threshold for all tests was set to $\mathrm{p}<0.05$. STATA software V.16 (StataCorp) was used for statistical analysis.

\section{Ethics}

The study was approved by the Danish Data Protection Agency (rec. no. 20/1681) and reported according to STROBE (Strengthening the Reporting of Observational Studies in Epidemiology) guidelines. ${ }^{26}$ Approval by ethical committee and informed consent was not necessary according to Danish legislation on medical ethics due to the register-based study design. ${ }^{27}$

\section{RESULTS}

Totally, 6624 patients ( $54.1 \%$ women) were discharged from the Department of Geriatric Medicine during the study period with a mean (SD) age of 83.0 (8.8) years. Of these, 847 patients (women $56.1 \%$ ) with a mean (SD) age 84.2 (8.3) years were discharged to the SNF (-OGT: $\mathrm{n}=440$ and+OGT: $\mathrm{n}=407$ ) (table 1 ), whereas 5777 patients (women 53.8\%) with a mean (SD) age of 82.9 (8.8) (preOGT $=3343$ and post-OGT $=2434)$ were discharged to their own home (non-SNF) (figure 1). Proportion of patients with $>1$ index admission was $58 \%$ (range 1-13).

\section{Characteristics and outcome data for SNF patients (-OGT and +0GT) ( $\mathrm{n}=847)$}

No difference was found between -OGT $(n=440)$ and +OGT $(n=407)$ regarding age, sex, BI, CGI and 30-day mortality. Median (IQR) LOS of index admission was $7.8(5.0-12.8)$ days and $6.0(3.9-10.0)$ days in -OGT and +OGT, respectively $(\mathrm{p} \leq 0.0001)$ (table 1$)$. The median number of OGT visits pr. patient was 1 ((IQR 1-2) range 1-10). The proportion of patients visited more than once was $38 \%$. In $32 \%$ of the visits the patients had adjustments to their medication, $14 \%$ had blood samples taken, $12 \%$ 
Table 1 Characteristics of patients discharged from department of geriatric medicine to a skilled nursing facility without follow-up (-OGT) or with follow-up (+OGT) by an OGT and patients not discharged to a skilled nursing facility from department of geriatric medicine before (pre-OGT) and after (post-OGT) implementation of OGT

\begin{tabular}{|c|c|c|c|c|c|c|}
\hline & -OGT $(n=440)$ & +OGT $(n=407)$ & $P$ value & $\begin{array}{l}\text { Pre-OGT } \\
(n=3343)\end{array}$ & $\begin{array}{l}\text { Post-OGT } \\
(n=2434)\end{array}$ & $P$ value \\
\hline Age, years, mean (SD) & $84.2(8.0)$ & $84.2(8.5)$ & 0.98 & $82.7(8.8)$ & $83.1(8.9)$ & 0.144 \\
\hline Female, n (\%) & $240(54.6)$ & 235 (57.9) & 0.329 & $1805(54.0)$ & $1307(53.7)$ & 0.817 \\
\hline Barthel Index, n (\%) & & & 0.6 & & & 0.012 \\
\hline $80-100$ & $12(2.8)$ & $9(2.2)$ & & $462(13.8)$ & 303 (12.5) & \\
\hline $50-79$ & $80(18.7)$ & $71(17.4)$ & & $924(27.6)$ & $655(27.0)$ & \\
\hline $25-49$ & $121(28.3)$ & $117(28.8)$ & & $716(21.4)$ & $600(24.7)$ & \\
\hline $0-24$ & $215(50.2)$ & $191(46.9)$ & & $1083(32.4)$ & $738(30.3)$ & \\
\hline Missing & $12(2.7)$ & $19(4.7)$ & & $158(4.7)$ & $129(5.6)$ & \\
\hline $\mathrm{CCl}$, median (IQR) & $2(1-3)$ & $2(1-3)$ & 0.182 & $2(1-4)$ & $2(1-3)$ & 0.146 \\
\hline CCI, n (\%) & & & 0.37 & & & 0.45 \\
\hline 0 & $93(21.1)$ & 99 (24.3) & & $682(20.4)$ & $521(21.4)$ & \\
\hline $1-2$ & $165(37.5)$ & $157(38.6)$ & & $1233(36.9)$ & $911(37.4)$ & \\
\hline$\geq 3$ & $182(41.4)$ & $151(37.1)$ & & $1428(42.7)$ & $1002(41.2)$ & \\
\hline LOS, days (index admission) median (IQR) & $7.8(5.0-12.8)$ & $6.0(3.9-10.0)$ & 0.0001 & $5.1(3.1-7.9)$ & $4.8(2.9-6.9)$ & 0.0001 \\
\hline Died within 30 days after discharge, $n(\%)$ & $75(17.1)$ & $68(16.7)$ & 0.896 & $360(10.8)$ & $233(9.6)$ & 0.139 \\
\hline
\end{tabular}

$\mathrm{CCl}$, Charlson Comorbidity Index; LOS, length of stay; OGT, outgoing geriatric team.

were given nutritional advice, $5 \%$ were informed of tests results (ie, X-ray, endoscopy) and 6\% received intervention from a specialised municipal acute nursing function.

The 30-day readmission rate declined from start of intervention from $36.8 \%(\mathrm{n}=162)$ in -OGT to $27.8 \%$ $(\mathrm{n}=113)$ in +OGT group $(\mathrm{p}=0.005)$ (figure 2$)$. The cumulative incidence of readmission $(95 \% \mathrm{CI})$ was $39.8 \%$ (35.2 to 44.8 ) in the -OGT group and $30.2 \%$ (25.8 to 35.2 ) in +OGT group (figure 3). Unadjusted risk (HR $(95 \% \mathrm{CI}))$ of readmission was 0.68 ( 0.54 to $0.87, \mathrm{p}=0.002)$ in the +OGT group compared with the -OGT group. Risk of readmission remained lower in the +OGT group in the fully adjusted model (0.72 (0.57 to 0.93), $\mathrm{p}=0.011$ ). Subgroup analysis defining readmission as an unplanned hospital contact with a duration of 12 +hours, occurring
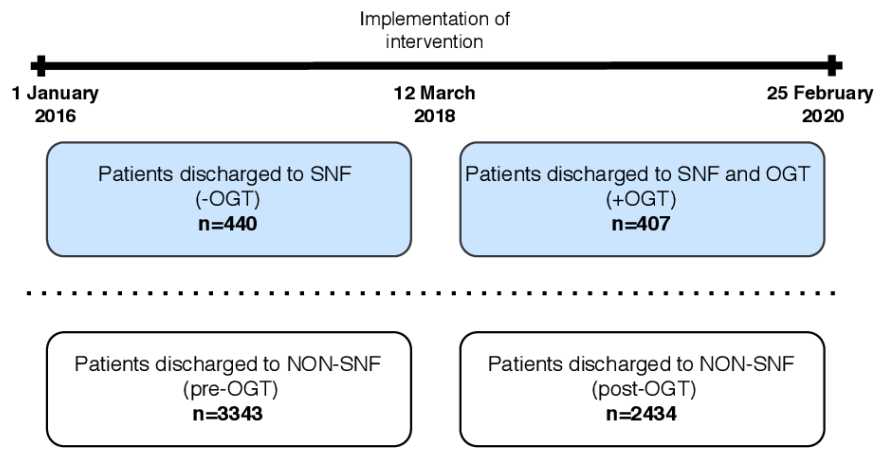

Figure 1 Timeline of patients discharged from the department of geriatric medicine according to discharge destination and initiation of OGT. OGT; outgoing geriatric team, pre-OGT; before implementation of OGT, post-OGT; after implementation of OGT, SNF; skilled nursing facility. between 4 hours and 30 days after discharge from the Department of Geriatric Medicine did not change the results (data not shown).

Patients had a wide spectrum of primary diagnoses at index admission and readmission with no difference between the two groups in regards of proportions within each ICD-10 groups $\left(\chi^{2}\right)$. The three most common ICD-10 groups were diseases of the respiratory organs (ICD10: J00-J99) (-OGT: 27\% and +OGT: 22\%, $\mathrm{p}=0.127$ ); endocrine, nutritional and metabolic diseases (ICD10: E00E90) (-OGT: $10 \%$ and +OGT: $12 \%, p=0.271)$; and certain infectious and parasitic diseases (ICD10: A00-B99) (OGT: 9\% and +OGT: 9\%, $\mathrm{p}=0.996)$. The three most common ICD-10 groups at readmission were diseases of the respiratory organs (ICD10: J00-J99) (-OGT: 29\% and +OGT: $17 \%, \mathrm{p}=0.069)$; injury, poisoning and certain

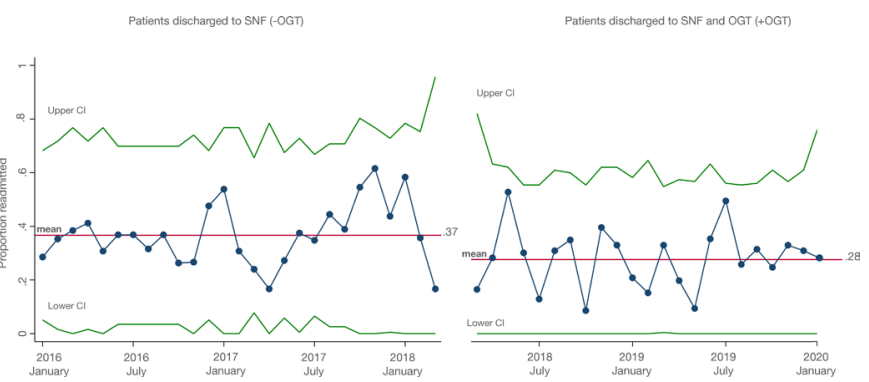

Figure 2 A statistical process control chart of monthly readmission rates during the study period among patients discharged to skilled nursing facility and OGT compared with patients discharged to skilled nursing facility only. OGT, outgoing geriatric team; SNF, skilled nursing facility. 


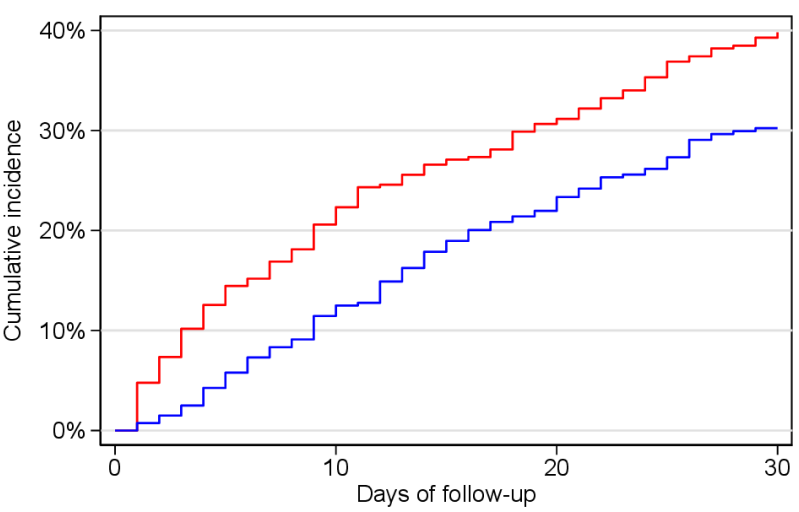

Number at risk$$
\text { -OGT } 440
$$

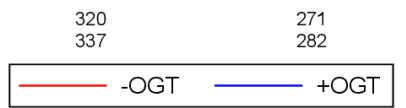

Figure 3 Kaplan-Meier curves of cumulative incidence of 30-day readmissions for any unplanned readmission for patients discharged to skilled nursing facility and OGT compared with patients discharged to skilled nursing facility only. OGT, outgoing geriatric team.

other consequences of external causes (ICD10: S00-T98) (-OGT: $11 \%$ and +OGT: 12\%, $\mathrm{p}=0.834$ ); and diseases of the circulatory system (ICD10: I00-I99) (-OGT: 10\% and +OGT: $11 \%, \mathrm{p}=0.692)$.

\section{Characteristics and outcome data for non-SNF patients (pre- OGT and post-0GT) $(n=5777)$}

Nodifferenceinage,sex,CCIand30-daymortalitywasfound between the pre-OGT $(n=3343)$ and post-OGT $(n=2434)$ groups, while the distribution of BI in the four subcategories differed significantly between groups $(\mathrm{p}=0.012)$. BI $80-100$ : pre-OGT $=13.8 \%$ and post-OGT $=12.5 \%$; BI 50-79: pre-OGT $=27.6 \%$ and post-OGT $=27.0 \%$; BI 25-49: pre-OGT $=21.4 \%$ and post-OGT $=24.7 \%$; BI $0-24$ : preOGT $=32.4 \%$ and post-OGT $=30.3 \%$. Median (IQR) LOS of index admission was 5.1 (3.1-7.9) days and 4.8 (2.9$6.9)$ days in pre-OGT and post-OGT groups, respectively $(\mathrm{p}<0.0001)$ (table 1$)$.

Among the pre-OGT and post-OGT groups the 30 days readmission rate was $26.5 \%(\mathrm{n}=887)$ and $27.8 \%(\mathrm{n}=676)$ $(\mathrm{p}=0.295)$, respectively. The cumulative incidence of readmission $(95 \% \mathrm{CI})$ was $29.1 \%$ (27.5 to 30.7$)$ in preOGT and $28.8 \%$ (27.0 to 30.7 ) in post-OGT (figure 4 ). No difference was found in the risk of readmission between the pre-OGT and post-OGT group, neither for unadjusted nor adjusted risk (HR $(95 \% \mathrm{CI})$ ) of 1.00 (0.90 to1.10, $\mathrm{p}=0.922$ ) and 1.01 (0.91 to $1.11, \mathrm{p}=0.920)$, respectively.

\section{DISCUSSION}

This study shows that follow-up visits by an OGT reduces hospital readmissions among patients discharged from a geriatric department to an SNF. The results remained significant even after adjusting for sex, age, in hospital LOS, comorbidity and functional status. Further, the

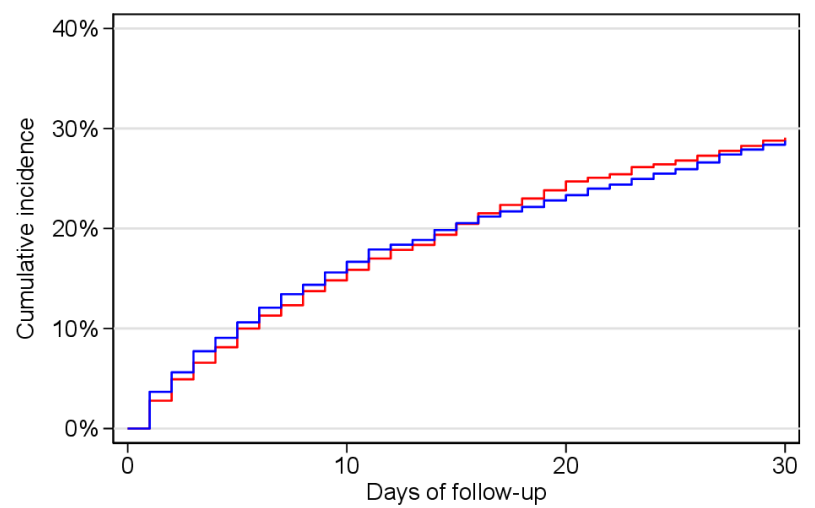

Number at risk

pre-OGT 3341

post-OGT 2425

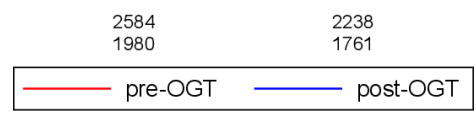

Figure 4 Kaplan-Meier curves of cumulative incidence of 30-day readmissions for any unplanned readmission for patients discharged to own home. Before-and-after implementation of OGT (pre-OGT and post-OGT). OGT, outgoing geriatric team.

effect of the intervention was immediate and persistent throughout the study period.

To our knowledge, only few other studies have assessed interventions to prevent readmission among patients discharged to SNF. ${ }^{4-6} 19$ A retrospective study from Cleveland, USA demonstrated a significant reduction in readmission rates from $28 \%$ to $22 \%(\mathrm{p}<0.001)$ after implementation of a connected care model. ${ }^{6}$ The applied model was very extensive with patients receiving visits from an outgoing team including doctors and nurses $4-5$ times a week after discharge from hospital to SNF and telephone coverage at nights, weekends and monthly meetings with multidisciplinary teams. This extensive model may be difficult to apply in other settings. Another US-study explored whether readmission could be reduced by implementing video conference to improve transition between hospital and an SNF. ${ }^{5}$ Videoconference reduced the 30-day readmission rate from $24 \%$ to $15 \%$ (OR $0.57,95 \%$ CI 0.34 to $0.96, \mathrm{p}=0.04$ ). However, the prospective cohort study compared preintervention and postintervention rates in two different SNF's, and the effect was mainly due to an increase of readmission in the control cohort rather than a reduction of readmissions in the intervention group. ${ }^{5}$ Interpretation and application of these studies' results to a different healthcare system, as the Danish, should be done with care, as large organisational variations exist across countries. A Danish randomised controlled trial studied the effect of comprehensive geriatric care offered to patients 65 years and older, referred from any hospital department to a rehabilitation unit. ${ }^{19}$ The study did not show any reduction in 90 days hospital readmission rates in the intervention group compared with usual care. However, the study did not explore 30-day readmission rates specifically and the negative result may be due to a spill over effect, that is, the intervention may have affected the control group, and a 
non-real-life setting since the intervention was performed by a single geriatrician only. ${ }^{19}$

Among the SNF patients in our study, the preintervention 30-day readmission rate was $36 \%$, which is high compared with an overall readmission rate of $18 \%$ among older hospitalised patients in Denmark. ${ }^{28}$ However, it is well known that patients discharged to SNF or other postacute care facilities have a higher risk of readmission. A study of patients discharged to postacute care facility demonstrated a readmission rate of $22 \%$ and most readmissions $(80 \%)$ occurred within 30 days of discharge. ${ }^{28}$ Other studies on patients discharged to temporary care have shown 30 -day readmission rates between $24 \%$ and $28 \%{ }^{4-6}$ This variation in readmission rates between studies may be explained by differences in patient characteristics, since other studies have included all residents at an SNF including residents referred to the SNF from home or a surgical department. Our study solely included the most vulnerable patients discharged from a geriatric department, characterised by high age, low functional status and multimorbidity and therefore at highest risk of readmission. ${ }^{8-10}$ Moreover, there may be differences in the definition of readmission. ${ }^{29}$ Our definition included any unplanned hospital contact within 30 days of discharge, thereby including brief contacts to the emergency department.

In-hospital LOS declined significantly during the study period. However, this decline was not reflected by a change in disease burden since CCI or primary diagnoses of hospital admission remained unchanged. The trend towards shorter hospital stay over time seen in our study is also reported on a national and European basis. ${ }^{30}$ In our study, LOS did not affect the readmission rate. The decrease in LOS was higher among patients discharged to SNF compared with patients not discharged to SNF. This indicates that the intervention may have affected LOS. The decision of when to discharge is based on the geriatrician's clinical judgement, but may have been affected by a knowledge of an OGT follow-up shortly after discharge, enabling patients to earlier discharge. However, the municipality decides, based on availability whether care and rehabilitation is provided at the SNF or at home. The geriatricians are seldom aware of this decision when discharge is planned. We, therefore, consider it less likely, that the intervention has affected LOS. However, other non-identified factors may have had an impact on the ability of SNF-patients to be discharged sooner. This must be addressed in future studies.

We found no difference in the proportion of men and women, which is surprising, since other studies of similar geriatric cohorts have shown a higher proportion of women compared with men. ${ }^{31}$ However, our sex ratio corresponds to the sex ratio shown in the Danish national database of geriatrics 2019. ${ }^{32}$

\section{Strengths and limitations of this study}

Our study has limitations. First, this was a before-and-after study, which has a risk of overestimating the effect ${ }^{33}$ due to residual confounding. The participants in the intervention group (+OGT) may have been exposed to other non-identified factors compared with the control group. However, readmission rates among non-SNF patients remained unchanged in the pre-OGT and post-OGT groups highlighting that no general change in the pattern of admissions occurred during the study period. Second, this was a single centre study, which limits the generalisability of study results. In addition, as no consensus definition of SNF exists, other studies may represent differently organised SNF's, dissimilar patient populations and other discharge procedures from geriatric departments worldwide, also limiting the generalisability. Third, the study only included data on hospital readmissions at Odense University Hospital, which may underestimate the risk of readmissions. However, only patients, who would have travelled or moved to other municipalities outside Odense, would be at risk of readmission to other hospitals than Odense University Hospital. In our dataset, we were able to track all patients, and none had residence outside the municipality of Odense within 30 days after discharge.

The study also has several strengths. We used data from registers with no patients lost to follow-up. In addition, we performed power and sample size calculation when planning the study to ensure appropriate LOS period. The adjusted analysis involved patient characteristics including measures of ADLs (Barthel Index), comorbidity (CCI) and LOS, which are important risk factors for readmission. ${ }^{8-10}$ Furthermore, our results are strengthened by accounting for the competing risk of death in our analysis, censoring those who died with-in 30 days of discharge. Finally, the study was carried out in a real-life setting and therefore implementable in similar settings.

Several elements of our OGT-intervention may have been crucial in the prevention of readmissions. The OGT facilitated a close co-operation between hospital, patient and SNF, which potentially prevented miscommunication and loss of information in the transition from secondary to primary care sector. Furthermore, early detection and correction of ambiguities and inadequate hospital care plans may have led to improved and shared goals of care for the benefit of the patient. A shared responsibility of the patient in the early days after discharge may have ensured confidence among patients, relatives and caregivers and prevented unnecessary contacts to doctors on call or emergency doctors. Lastly, reassessment of the patient's medical condition may have led to early detection and treatment of recurrence of disease, thereby preventing readmission.

Despite the potential benefit of outgoing hospital teams, the intervention is costly and hospital resources are redistributed outside the hospital. In our setup, limiting the postdischarge follow-up visit to patients discharged to SNF reduced the time expenditure since all patients were discharged to the same SNF and therefore the intervention may have been economical rentable. Cost-effective analysis of the intervention remains to be assessed before 
dissemination of this model. Our study does not give insight to subelements of the intervention, but further studies with an explorative or qualitative design should address this. Such insight may be valuable in the development of less costly interventions, such as telemedicine. Telemedicine and videoconference are likely to be less costly and perhaps as effective tools for posdischarge follow-up, which calls for further exploration.

\section{CONCLUSION}

Follow-up visits by an OGT significantly reduced 30-day readmission rates by $28 \%$ in older vulnerable patients recently discharged from hospital to an SNF.

\section{Author affiliations \\ ${ }^{1}$ Geriatric Research Unit, Department of Clinical Research, University of Southern Denmark, Odense, Syddanmark, Denmark \\ ${ }^{2}$ Department of Geriatric Medicine, Odense University Hospital, Odense, Denmark ${ }^{3}$ Department of Cross-sectoral Collaboration, Region of Southern Denmark, Vejle, Denmark \\ ${ }^{4}$ Department of Public Health, University of Southern Denmark, Odense, Syddanmark, Denmark}

Acknowledgements We wish to thank Rolf Kjærby (Odense University Hospital) and Rune Pedersen (Region of Southern Denmark) for their assistance in retrieving and merging data from the respective electronic patient journals and administrative systems, and Eva Sandberg and Inge Pedersen (OPEN-Open Patient data Explorative Network, Odense University Hospital, Region of Southern Denmark) for statistical counselling. A special thanks to the personnel at Lysningen (Odense Municipality) and the Department of Geriatric Medicines (Odense University Hospital) for their commitment and willingness to implement the outgoing geriatric function and cross-sectoral collaboration.

Contributors KT is the guarantor of the study. KT and AF directed and contributed equally to all aspects of this manuscript. KA-R, JR and LEM contributed to study design, data interpretation and writing. All authors have commented, read and approved the final manuscript.

Funding The Region of Southern Denmark and Odense University Hospital funded the study (14-17636).

Competing interests None declared.

Patient and public involvement Patients and/or the public were not involved in the design, or conduct, or reporting, or dissemination plans of this research.

Patient consent for publication Not required.

Provenance and peer review Not commissioned; externally peer reviewed.

Data availability statement Data are available on reasonable request.

Open access This is an open access article distributed in accordance with the Creative Commons Attribution Non Commercial (CC BY-NC 4.0) license, which permits others to distribute, remix, adapt, build upon this work non-commercially, and license their derivative works on different terms, provided the original work is properly cited, appropriate credit is given, any changes made indicated, and the use is non-commercial. See: http://creativecommons.org/licenses/by-nc/4.0/.

\section{ORCID iDs}

Katja Thomsen http://orcid.org/0000-0002-0454-836X

Anders Fournaise http://orcid.org/0000-0002-4754-7500

Jesper Ryg http://orcid.org/0000-0002-8641-3062

\section{REFERENCES}

1 Clegg A, Young J, Iliffe S, et al. Frailty in elderly people. Lancet 2013;381:752-62.

2 Creditor MC. Hazards of hospitalization of the elderly. Ann Intern Med 1993;118:219-23.
3 Hubbard RE, Peel NM, Samanta M, et al. Frailty status at admission to hospital predicts multiple adverse outcomes. Age Ageing 2017;46:801-6

4 Yoo JW, Jabeen S, Bajwa T, et al. Hospital readmission of skilled nursing facility residents: a systematic review. Res Gerontol Nurs 2015;8:148-56.

5 Moore AB, Krupp JE, Dufour AB, et al. Improving transitions to Postacute care for elderly patients using a novel Video-Conferencing program: ECHO-Care transitions. Am J Med 2017;130:1199-204.

$6 \mathrm{Kim}$ LD, Kou L, Hu B, et al. Impact of a connected care model on 30 -day readmission rates from skilled nursing facilities. J Hosp Med 2017;12:238-44.

7 Fournaise A, Espensen N, Jakobsen S, et al. Increasing primary health-care services are associated with acute short-term hospitalization of Danes aged 70 years and older. Eur Geriatr Med 2017;8:435-9.

8 Bahrmann A, Benner L, Christ M, et al. The Charlson comorbidity and Barthel index predict length of hospital stay, mortality, cardiovascular mortality and rehospitalization in unselected older patients admitted to the emergency department. Aging Clin Exp Res 2019;31:1233-42.

9 Pedersen MK, Meyer G, Uhrenfeldt L. Risk factors for acute care hospital readmission in older persons in Western countries: a systematic review. JBI Database System Rev Implement Rep 2017;15:454-85.

10 Wang P, Wang Q, Li F, et al. Relationship between potentially inappropriate medications and the risk of hospital readmission and death in hospitalized older patients. Clin Interv Aging 2019;14:1871-8.

11 Lembeck MA, Thygesen LC, Sørensen BD, et al. Effect of single follow-up home visit on readmission in a group of frail elderly patients - a Danish randomized clinical trial. BMC Health Serv Res 2019;19:751.

12 Lindegaard Pedersen J, Pedersen PU, Damsgaard EM. Nutritional Follow-Up after Discharge Prevents Readmission to Hospital - A Randomized Clinical Trial. J Nutr Health Aging 2017;21:75-82.

13 Lisby M, Klingenberg M, Ahrensberg JM, et al. Clinical impact of a comprehensive nurse-led discharge intervention on patients being discharged home from an acute medical unit: randomised controlled trial. Int J Nurs Stud 2019;100:103411.

14 Thygesen LC, Fokdal S, Gjørup T, et al. Can municipality-based post-discharge follow-up visits including a general practitioner reduce early readmission among the fragile elderly $(65+$ years old)? A randomized controlled trial. Scand J Prim Health Care 2015;33:65-73.

15 Finlayson K, Chang AM, Courtney MD, et al. Transitional care interventions reduce unplanned Hospital readmissions in high-risk older adults. BMC Health Serv Res 2018;18:956.

16 Hansen LO, Young RS, Hinami K, et al. Interventions to reduce 30-day rehospitalization: a systematic review. Ann Intern Med 2011;155:520-8.

17 Renaudin P, Boyer L, Esteve M-A, et al. Do pharmacist-led medication reviews in hospitals help reduce hospital readmissions? A systematic review and meta-analysis. Br J Clin Pharmacol 2016;82:1660-73.

18 Berkowitz RE, Fang Z, Helfand BKI, et al. Project reengineered discharge (red) lowers Hospital readmissions of patients discharged from a skilled nursing facility. J Am Med Dir Assoc 2013;14:736-40.

19 Zintchouk D, Gregersen M, Lauritzen T, et al. Geriatrician-performed comprehensive geriatric care in older adults referred to an outpatient community rehabilitation unit: a randomized controlled trial. Eur $J$ Intern Med 2018;51:18-24.

20 Gardner RL, Pelland K, Youssef R, et al. Reducing hospital readmissions through a skilled nursing facility discharge intervention: a pragmatic trial. J Am Med Dir Assoc 2020;21:508-12.

21 Vrangbaek K. Denmark. In: Tikkanen R, Osborn R, Mossialos E, eds. International health care system profiles: the Commonwealth fund, 2020.

22 Esbjerg municipality, Copenhagen municipality, Aarhus municipality. The 6-cities key figures 2019 [6-by nøgletal 2019], 2019. Available: https://www.odense.dk/om-kommunen/statistikker-og-regnskaber/ odense-i-tal/6-by-nogletal [Accessed 20 Sep 2020].

23 authorities TDh. Description of indicators - natiolnal aim [Beskrivele af indikatorer - de nationale mål, 2016.

24 Matzen LE, Jepsen DB, Ryg J, et al. Functional level at admission is a predictor of survival in older patients admitted to an acute geriatric unit. BMC Geriatr 2012;12:32.

25 Thygesen SK, Christiansen CF, Christensen S, et al. The predictive value of ICD-10 diagnostic coding used to assess Charlson comorbidity index conditions in the population-based Danish national Registry of patients. BMC Med Res Methodol 2011;11:83. 
26 Vandenbroucke JP, von Elm E, Altman DG, et al. Strengthening the reporting of observational studies in epidemiology (STROBE): explanation and elaboration. Int J Surg 2014;12:1500-24.

27 National Committee on Health Research Ethics. What to notify? 2020. Available: https://en.nvk.dk/how-to-notify/what-to-notify [Accessed 15 May 2020].

28 The Danish Health Authority. Health agremments, 2020. Available: https://www.esundhed.dk/home/emner/patienter\%20og\% 20sygehuse/sundhedsaftaler [Accessed 06 Jun 2020].

29 Kristensen SR, Bech M, Quentin W. A roadmap for comparing readmission policies with application to Denmark, England, Germany and the United States. Health Policy 2015;119:264-73.

30 European Observatory on Health Systems and Policies (OECD and WHO). State of health in the EU, Denmark, country health profile 2019, 2019.
31 Ryg J, Engberg H, Mariadas P, et al. Barthel index at hospital admission is associated with mortality in geriatric patients: a Danish nationwide population-based cohort study. Clin Epidemiol 2018;10:1789-800.

32 Danish Clincal Quality Program [Regionernes Kliniske Kvalitetsudviklingsprogram]. Annual report for the Danish national Geriatric Database, 2019 [Årsrapport for den landsdækkende database for geriatri, 2019], 2019. Available: https://www.sundhed. dk/content/cms/9/4709_geriatri_aarsrapport2019_endelig_ anonymiseret.pdf [Accessed 15 Sep 2020].

33 William Shaddish R, Campbell T. Experimental and quasiexperimental designs for generalized causal inference. 2nd edn, 2001. 